\title{
A modified in situ RT-PCR method for localizing fungal-specific gene expression in Candida-infected mice renal cells
}

\begin{abstract}
In situ Reverse Transcriptase PCR (in situ RT-PCR) can amplify mRNA and localize gene expression in cells. However, this method is not feasible in fungi as the thick fungal cell wall constitutes a barrier to this procedure. We developed a two step in situ RT-PCR procedure which enabled the detection and localization of Candida tropicalis mRNA expression in formalin-fixed, paraffin-embedded (FFPE) mouse kidney sections. This in situ hybridization study revealed the first direct evidence for deposition of Candida tropicalis secreted aspartic proteinase 2 (CtSAP2) in the tip of pseudohyphae and its involvement in acute systemic candidiasis. We conclude that in situ RT-PCR can be successfully applied to FFPE tissues and will offer new perspectives in studying gene expression in Candida species.
\end{abstract}

Keyword: Candida tropicalis; In situ RT-PCR; Secreted aspartyl proteinase; Biotinylated dUTP; Gene expression 